\title{
Analysis of pediatric dermatology inpatient consultations in a pediatric teaching hospital
}

\author{
Fatma S. Afsar, M.D. ${ }^{a, b}$
}

\begin{abstract}
Introduction. Although skin diseases are associated with low rate of hospitalization, dermatological manifestations are frequent in hospitalized patients. The aim of the study was to describe the inpatient dermatological consultations in a pediatric teaching hospital.

Population and Methods. Recorded data from inpatient pediatric dermatology consultation requests on a total of 539 consecutive inpatients (aged 0-18 years) from January 2004 to April 2010 were analyzed for consult diagnosis, dermatological disease group, primary diagnosis, requesting department, diagnostic pattern, treatment modality and referral to another department.
\end{abstract}

Results. Of the 539 inpatients, 310 (57.51\%) were males and $229(42.49 \%)$ were females. The most frequent requesting department was general pediatrics $(37.5 \%)$ followed by oncology $(15.6 \%)$ and pediatric surgery $(11.1 \%)$. Most of the patients $(32.1 \%)$ had been hospitalized for dermatological or related disease followed by acute lymphoblastic leukemia $(4.1 \%)$, chronic renal failure $(2.6 \%)$, bronchopneumonia $(2.6 \%)$ and epilepsy $(2.4 \%)$. Allergic skin diseases $(47.1 \%)$ were the leading group of dermatoses, which were followed by infectious diseases $(14.7 \%)$, and systemic diseases with cutaneous manifestations $(10.2 \%)$. Atopic dermatitis $(7.4 \%)$ and unclassified eczema $(7.4 \%)$ were the most frequent dermatoses followed by papular urticaria $(5.4 \%)$. Most of the patients $(80.0 \%)$ had diagnosis on clinical basis, whereas skin biopsy was performed in $15.9 \%$ and laboratory investigation in $4.1 \%$ of the patients. Local treatment was applied to $50.8 \%$ of the patients, systemic treatment to $5.8 \%$, local and systemic treatment together to $31.0 \%$ while no treatment was given to $11.1 \%$. Only $1.9 \%$ of the patients were referred to another department.

Conclusions. This study provided important data on the spectrum of skin disorders and their management in pediatric inpatients from the consultation perspective in a pediatric teaching hospital with multispecialty clinics.

Key words: consultation, inpatients, pediatrics, dermatology.

http:/ / dx.doi.org/10.5546/ aap.2017.eng.e377

To cite: Afsar FS. Analysis of pediatric dermatology inpatient consultations in a pediatric teaching hospital. Arch Argent Pediatr 2017;115(6):e377-e384.

\section{INTRODUCTION}

Majority of dermatological practice is done in an outpatient setting, but there are some conditions where children have to be hospitalized for skin disease or secondary systemic illness. ${ }^{1,2}$ The pediatricians are usually the first contact with the patients. After hospitalization referrals are given to different departments. ${ }^{3}$

Although cutaneous complaints contribute about one-third of all consultations in pediatric practice, there are only a few studies describing the inpatient consultation requests in pediatric dermatology (PD) and their effects on inpatient management. ${ }^{1,3-7}$ The aim of this study was to describe the PD inpatient consultations in a pediatric teaching hospital.

\section{POPULATION AND METHODS}

Five hundred thirty nine consecutive requests for PD inpatient consultation from January 2004 to April 2010 in Dr. Behcet Uz Children's Hospital were retrospectively evaluated for final dermatological consult diagnosis, dermatological disease group, primary diagnosis for hospital admission, requesting department, diagnostic procedure, treatment modality, sort of infection if present and need for another consultation to another department. The institution is a pediatric teaching hospital with 368 beds making it one of the biggest pediatric medical centers in Turkey since it was established in 1947.

The patients were divided into five different age groups: infant $(0-2$ years); preschool children (3-5 years); primary school children (6-10 years); preadolescent (11-12 years); and adolescent (13-18 years).

All patients were seen by the 
author in the same day of the request within a few hours. The final consult diagnoses and primary diagnoses for hospital admission were recorded. The dermatological disease groups were determined partly based on the Textbook of Pediatric Dermatology. ${ }^{8}$ The dermatological diagnoses that were not distinctively under a disease group were categorized as miscellaneous. Diagnoses were based on clinical features, skin biopsy and relevant laboratory investigations.

All data collected were analyzed using the descriptive statistics (SPSS version 16.0 Chicago, IL, USA).

\section{RESULTS}

Among the total of 539 pediatric inpatients for whom PD consultation was requested, 310 $(57.51 \%)$ were males and $229(42.49 \%)$ were females with a mean age of $5.32(+4.74)$ ranging from 0 to 18 years (male:female ratio 1.3:1).

TABLE 1. The most frequent 10 skin diseases established in all pediatric dermatology inpatient consultations

\begin{tabular}{lcc}
\hline Skin diseases & $\begin{array}{c}\text { Inpatients } \\
\text { (n) }\end{array}$ & $\begin{array}{c}\text { (\% within all } \\
\text { consultations) }\end{array}$ \\
\hline Atopic dermatitis & 40 & $(7.4 \%)$ \\
Unclassified eczema & 40 & $(7.4 \%)$ \\
Papular urticarial & 29 & $(5.4 \%)$ \\
Irritant contact dermatitis & 28 & $(5.2 \%)$ \\
Henoch Schönlein vasculitis & 26 & $(4.8 \%)$ \\
Diaper dermatitis & 24 & $(4.5 \%)$ \\
Seborrheic dermatitis & 20 & $(3.7 \%)$ \\
Maculopapular drug eruption & 18 & $(3.3 \%)$ \\
Pyoderma & 17 & $(3.2 \%)$ \\
Urticaria & 16 & $(3.0 \%)$ \\
\hline
\end{tabular}

TABLE 2. The most frequent 12 primary reasons (diagnoses) for hospital admission in all pediatric dermatology inpatient consultations

\begin{tabular}{lcc}
\hline $\begin{array}{l}\text { Primary reason for } \\
\text { hospital admission }\end{array}$ & $\begin{array}{c}\text { Inpatients } \\
\text { (n) }\end{array}$ & $\begin{array}{c}\text { (\% within all } \\
\text { consultations) }\end{array}$ \\
\hline Dermatologic symptom or & 173 & $(32.1 \%)$ \\
related disease & 96 & $(17.8 \%)$ \\
Unknown & 22 & $(4.1 \%)$ \\
Acute lymphoblastic leukemia & 14 & $(2.6 \%)$ \\
Chronic renal failure & 14 & $(2.6 \%)$ \\
Bronchopneumonia & 13 & $(2.4 \%)$ \\
Epilepsy & 10 & $(2.2 \%)$ \\
Acute myeloid leukemia & 8 & $(1.9 \%)$ \\
Aganglionic megacolon & 7 & $(1.5 \%)$ \\
Septicemia & 7 & $(1.3 \%)$ \\
Arthritis & 7 & $(1.3 \%)$ \\
Metabolic disease & 7 & $(1.3 \%)$ \\
Thalassemia major & &
\end{tabular}

Two hundred fifteen $(39.9 \%)$ of the patients were infants, $91(16.9 \%)$ were preschool children, $130(24.1 \%)$ were primary school children, 41 $(7.6 \%)$ were preadolescents, and $62(11.5 \%)$ were adolescents.

A total of 112 skin diseases were observed in 539 pediatric inpatients, which were categorized under 10 dermatological skin disease groups (see Annex). Allergic skin diseases established in $254(47.1 \%)$ inpatients was the leading group of dermatoses followed by infectious skin diseases in $79(14.7 \%)$ and systemic disorders with cutaneous manifestations in $55(10.2 \%)$. Atopic dermatitis (AD) established in $40(7.4 \%)$ inpatients and unclassified eczema in $40(7.4 \%)$ were the most common dermatoses followed by papular urticaria in $29(5.4 \%)$ and irritant contact dermatitis in $28(5.2 \%)$. The frequencies of cutaneous viral infections and bacterial infections were equal, each in $32(5.9 \%)$ inpatients followed by fungal infections in $12(2.2 \%)$ inpatients and parasitic infections in $3(0.6 \%)$ inpatients. The most frequent 10 skin diseases established in all pediatric inpatients are listed in Table 1.

A total of 96 diseases were defined as primary diagnosis for hospital admission including dermatological symptom or related disease in 539 pediatric inpatients. Dermatological symptom or related disease was the leading primary reason for hospital admission for 173 $(32.1 \%)$ inpatients followed by lymphoblastic leukemia for $22(4.1 \%)$, chronic renal failure for 14 $(2.6 \%)$ and bronchopneumonia for $14(2.6 \%)$. The primary diagnosis was not specified for $96(17.8 \%)$ inpatients in the consultation request forms. The most frequent 12 primary diagnoses for hospital admission in all PD inpatient consultations are listed in Table 2.

TABle 3. Distribution of pediatric dermatology inpatient consultations by requesting departments

\begin{tabular}{lcc}
\hline Requesting department & Inpatients (n) & $(\mathbf{\%})$ \\
\hline General Pediatrics & 202 & $(37.5 \%)$ \\
Hemotology-Oncology & 98 & $(18.1 \%)$ \\
Pediatric surgery & 60 & $(11.1 \%)$ \\
Newborn Unit & 49 & $(9.1 \%)$ \\
Emergency Department & 44 & $(8.2 \%)$ \\
Infectious Diseases & 44 & $(8.2 \%)$ \\
Cardiology & 16 & $(2.9 \%)$ \\
Hemodialysis Unit & 10 & $(1.9 \%)$ \\
Orthopedics and Traumatology & 7 & $(1.3 \%)$ \\
Intensive Care Unit & 6 & $(1.1 \%)$ \\
Plastic and Reconstructive Surgery & 3 & $(0.6 \%)$ \\
\hline Total & $\mathbf{5 3 9}$ & $\mathbf{( 1 0 0 . 0 \% )}$ \\
\hline
\end{tabular}


The most common requesting department was general pediatrics (GP) for $202(37.5 \%)$ consultations followed by Hematology-Oncology (H-O) for 98 (18.1\%), pediatric surgery (PS) for $60(11.1 \%)$ and newborn unit (NU) for $49(9.1 \%)$. Distribution of PD inpatient consultations by the requesting departments are summarized in Table 3.

Of the 539 consultations, $431(80.0 \%)$ diagnoses were based on clinical assessment, The distribution of diagnostic procedures for consultations are listed in Table 4. Topical treatment alone was the leading treatment pattern applied to $274(50.8 \%)$ inpatients, followed by systemic treatment together with topical treatment applied to $167(31.0 \%)$ inpatients, systemic treatment alone applied to $31(5.8 \%)$ inpatients, other treatments (percutaneous abscess drainage, curettage for removal of skin lesions, medical dressing, etc.) alone applied to four $(0.7 \%)$ inpatients, systemic treatment and other treatments together applied to two $(0.3 \%)$ inpatients, and topical treatment and other treatments (percutaneous abscess drainage, curettage for removal of skin lesions, medical dressing, etc.) together applied to one $(0.2 \%)$ inpatient. Sixty $(11.1 \%)$ inpatients had no treatment. Ten $(1.8 \%)$ inpatients were referred to another department for finalization of the consultation (Table 5). The reasons for referrals were surgical excision in seven $(7 / 10)$ inpatients, further investigation for systemic involvement in one $(1 / 10)$ patient, and other reasons in two $(2 / 10)$ inpatients.

Table 4. Diagnostic procedures for the pediatric dermatology inpatient consultations

\begin{tabular}{lcc}
\hline Type of diagnostic procedure & Inpatients (n) & $\mathbf{( \% )}$ \\
\hline Clinical diagnosis & 431 & $(80.0 \%)$ \\
Skin biopsy & 86 & $(15.9 \%)$ \\
Laboratory investigation & 22 & $(4.1 \%)$ \\
\hline Total & $\mathbf{5 3 9}$ & $\mathbf{( 1 0 0 . 0 \% )}$ \\
\hline
\end{tabular}

TABLE 5. The departments to which referrals were made for finalization of the consultations

\begin{tabular}{lcc}
\hline Departments & Inpatients (n) & $\mathbf{( \% )}$ \\
\hline Plastic and Reconstructive Surgery & 4 & $(0.7 \%)$ \\
Otorhinolaryngology & 2 & $(0.4 \%)$ \\
Orthopedics and Traumatology & 2 & $(0.4 \%)$ \\
Pediatric surgery & 1 & $(0.2 \%)$ \\
Pediatric neurology & 1 & $(0.2 \%)$ \\
\hline Total & $\mathbf{1 0}$ & $\mathbf{( 1 0 0 . 0 \% )}$ \\
\hline
\end{tabular}

\section{DISCUSSION}

Skin related problems may be present at the time of hospitalization as a primary skin disease or they may develop or exacerbate during the stay in hospital, requiring dermatology consultation. ${ }^{3}$ Common dermatologic diseases are often not recognized or they are misdiagnosed by nondermatologists. ${ }^{1}$ Thus, inpatient consultation is the ideal environment to capture the different perceptions that other physicians have of patients and dermatologists. ${ }^{2}$ Although it has been suggested that the role of dermatologists as consultants for pediatric inpatients is similar to their role in outpatient consultations, reasons for dermatology consultation and the extent to which it contributes to cutaneous care are not well documented. ${ }^{7,9}$

In this study of PD inpatient consultations, there was a slight male preponderance with a male:female ratio of 1.3:1 which was compatible with other similar studies. ${ }^{3,5}$ Male gender has been described as a risk factor associated with hospitalization of children. ${ }^{10,11}$ Inpatient dermatology consultation was most frequently requested for the infantile group consistent with the results of other studies. ${ }^{3,5}$ The great predominance of hospitalizations during the first years of life shows that the immunological immaturity of those children and their anatomical characteristics put them at a higher risk of having a severe case disease and getting sick in general. Besides, considering the growing importance of perinatal diseases as causes of hospitalization it is natural that children younger than one year old are the most hospitalized group. ${ }^{10}$

Allergic skin diseases were the leading group of dermatoses followed by infectious skin diseases and systemic diseases with cutaneous manifestations. Although allergic skin diseases and infectious skin diseases constitute the largest number of diagnoses in outpatient PD, systemic diseases with cutaneous manifestations are responsible for a much less number of patients. ${ }^{12}$ This shows us that systemic diseases with cutaneous manifestations are rare in childhood, but if present they are really serious and sometimes life-threatening and hospitalization is necessary. Another study had found that the most common diagnostic groups were diseases of the skin and subcutaneous tissue and infections and parasitic diseases. ${ }^{7}$

Proportional to the fact of allergic skin diseases, AD and unclassified eczema were the most frequent dermatoses followed by 
papular urticaria and irritant contact dermatitis. Eczemas, including $\mathrm{AD}$, have a higher prevalence in developed countries, being influenced by socioeconomic and environmental factors. ${ }^{13} \mathrm{It}$ should be considered that prevalence of $\mathrm{AD}$ in children ranges from $15 \%$ to $20 \%$ and it was also the most frequently diagnosed disorder in outpatient PD series. ${ }^{12,14}$

Papular urticaria which was a common problem for pediatric inpatients had been reported to contribute to $5.27 \%$ of pediatric dermatoses. ${ }^{15}$ It may be frequent due to fact that most of the pediatric inpatients come from rural or semirural areas. ${ }^{12}$ They may also have it while staying in the hospital. Irritant contact dermatitis was the other frequent consult diagnosis in pediatric inpatients. Sweating, detergents, soaps used in vigorous washing of patients, antiseptics in general and specifically in surgical washing preparations, dressing occlusion, bed confinement, and monitoring with catheters or pressure tubes are factors that together can cause contact dermatitis during hospitalization. ${ }^{2}$

In the second leading disease group, infectious diseases, bacterial and viral infections were equally predominant. They were followed by fungal infections and a very small number of parasitic infections. Cutaneous mycobacterial infection was not seen in any of the pediatric inpatients. Pyoderma was the leading diagnosis in this group, followed by herpes simplex infection, impetigo, tinea capitis, verrucae, and varicella infection. This high frequency of infections in inpatients were explained by the immunosuppression of some patients and the presentation of skin infections as a common reason for patient admission. ${ }^{3,15,16}$ The cases of neonatal varicella, staphylococcal scalded skin syndrome, extensive bullous impetigo, congenital cutaneous candidiasis, cellulitis, necrotizing fasciitis, abscess which were the primary dermatological cases required hospitalization and regular monitoring both by the dermatologist and pediatrician in another study. Other skin infections were co-existing along with primary systemic complaints. ${ }^{3}$

One of the most challenging and rewarding aspects of inpatient dermatology consultation is the diagnosis of systemic disorders with cutaneous findings. The skin changes may be the first sign of the underlying condition, and rapid identification can have a significant impact and sometimes be life-saving. ${ }^{17}$ In this study, Henoch Schönlein vasculitis was the fifth most frequent diagnosis among all diagnoses, but other systemic disorders with cutaneous manifestations such as collagen vascular diseases, purpura fulminans or Behcet's disease were seen in minority of pediatric inpatients. Another common indication for PD referral was reported to be drug eruptions. ${ }^{6}$ Maculopapular drug eruption was among the first ten leading diagnoses, while fixed drug eruption was seen in a lower frequency in this study. These reactions can be explained by the large amounts of drugs received by inpatients during hospitalization and dermatology consultation is helpful in identifying the most likely responsible medication. ${ }^{5,16}$

Genodermatoses were the fourth most commonly diagnosed disease group in which epidermolysis bullosa was the most frequent one followed by ichtyoses probably due to frequent consanginous marriages. The disease group 'miscellaneous' came after the genodermatoses in frequency. In another study of PD inpatient consultations, over $40 \%$ of diagnoses were categorized as 'miscellaneous' emphasizing the need to 'expect the unexpected' ${ }^{5}$ The rate of miscellaneous group was low in this series.

Although diseases of hair, nails, sebaceous and eccrine glands were not very frequent among pediatric inpatients, nail dystrophy, acne, alopecia areata and scarring alopecia were the most frequent diagnoses. Most of these diagnoses may already present and they may be noticed during hospitalization. The rate of this group has been reported to be twice as much in PD outpatients. ${ }^{12}$

The group of 'erythemas and blistering diseases' was seventh in frequency in this series, but this rate was quite high when it was compared with the outpatient practice. ${ }^{12}$ This condition shows us that the disease group is quite serious and hospitalization is necessary for pediatric age groups. Erythema multiforme was the leading diagnosis followed by erythroderma and erythema nodosum in this group. Disorders of pigmentation / melanocyte and epidermal nevi are mostly handled in outpatient settings and their rate was quite low in inpatients. Vitiligo, congenital nevus and postinflammatory hypopigmentation were the most common diagnoses which were possibly present in inpatients before hospitalization.

Papulosquamous disorders constituted a small group in the inpatient consultation diagnoses. Psoriasis was not a common problem among pediatric inpatients, but its rate was close to the rates of reference outpatient series. ${ }^{12,14}$ However, 
it was the third most frequent diagnosis among pediatric inpatients in another study. ${ }^{6}$ The relative low rates of vascular and lymphatic anomalies, and especially hemangiomas in this study show us that these disorders are being handled in the outpatient setting. In contrast, vascular anomalies were reported to be one of the two most common diagnoses when hospitalization was primarily for skin related disease in another study of PD inpatient consultations. ${ }^{5}$

GP was the most frequent requesting department followed by H-O, PS and NU. The substantial number of requests by the departments of GP and PS correlates with the number of patients admitted. The heaviness of consultations from $\mathrm{H}-\mathrm{O}$ and $\mathrm{NU}$ may be due to fact that most of their patients should be followed in the hospital setting for their primary complaints. GP is the department from which the maximum number of consult requests were made in other studies. ${ }^{3,5,6}$ Also, GP was reported to be followed by pediatric $\mathrm{H}-\mathrm{O}$, pediatric intensive care unit, neonatology, child psychiatry, otorhinolaryngology and PS as the main consultrequesting services in descending order for the consultation request in one of those studies. ${ }^{6}$

Unlike other previous studies, the inpatients were evaluated for primary hospital admission reasons and it was found that almost one-third of them had been hospitalized for a dermatological or related disorder by the pediatricians. The following most frequent primary diagnoses were acute lymphoblastic leukemia, chronic renal failure, bronchopneumonia and epilepsy. The primary diagnoses were not defined in some of the consultation request forms. Those missing primary diagnoses may be related or unrelated to dermatological disorders.

Dermatologists are often able to make a clinical diagnosis without additional investigations which are sometimes ordered by other physicians. ${ }^{18}$ A great majority of diagnoses were found to be established on clinical basis in this series, while skin biopsy was performed in $15.9 \%$ of the inpatients. This rate was in concordance with other PD inpatient consultation series, but it was quite superior than reference outpatient series. ${ }^{3,5,12,14}$ This shows us that skin biopsy is much more essential for establishing a final and absolute diagnosis for inpatient consultations. The need for additional laboratory investigations was not more than the need in the outpatient setting. ${ }^{12}$

Data describing the management of pediatric inpatients for whom dermatology consultation was requested are limited. ${ }^{6}$ In this study, half of the pediatric inpatients were treated by topical treatments pointing out that topical treatment was the mainstay treatment for the inpatients similar to PD outpatients. ${ }^{12,14}$ Topical treatment was followed by topical and systemic treatment together, and medical advice or reassurance was given to nearly one-tenth of the inpatients. The overall rate of referral to another department for the finalization of consultations was as low as $1.8 \%$. A great number of those inpatients were proposed to be referred to surgical departments such as plastic and reconstrictive surgery and otorhinolaryngology, mainly for excisional surgery. It can be inferred that skin problems of the pediatric inpatients are easily resolved within PD consultations without a need for further consultations except for specific surgical procedures.

Additionally, the need for dermatology education of pediatricians is obvious. Most of the dermatological diseases presented by the inpatients were not the cause of hospitalization except the drug eruptions. Papular urticaria and contact dermatitis may also emerge during hospitalization. Training of pediatric dermatologists and pediatricians focus on accurate recognition, diagnosis, and management of these common skin diseases, ${ }^{19}$ Thus, PD consultation is an opportunity to acquire dermatological notion for them and pediatric dermatologist plays an important role in the diagnosis and treatment of pediatric skin disorders. In some programs, an inpatient consultation may be the only exposure a resident has to dermatology. ${ }^{17}$ The limitations of this study were its retrospective design which could result in incomplete data and poor documentation of the clinical symptoms and involvement of the inpatients who were evaluated by a single dermatologist.

\section{CONCLUSIONS}

A diverse spectrum of skin disorders established in PD inpatient consultations points out that failure to identify some of the inpatient cases can be quite serious. However, some of the inpatients may have skin disorders that can be handled in the outpatient practice. This study provided information that PD inpatient consultation has an important role in the management of inpatients in a pediatric teaching hospital with multispecialty clinics. 


\section{REFERENCES}

1. Falanga V, Schachner LA, Rae V, et al. Dermatologic consultations in the hospital setting. Arch Dermatol 1994;130(8):1022-5.

2. Peñate $Y$, Guillermo N, Melwani P, et al. Dermatologists in hospital wards: an 8-year study of dermatology consultations. Dermatology 2009;219(3):225-31.

3. Srinivas SM, Hiremagalore R, Venkataramaiah LD, et al. Pediatric dermatology inpatient consultations: a retrospective study. Indian J Pediatr 2015;82(6):541-4.

4. Schachner L, Ling NS, Press S. A statistical analysis of a pediatric dermatology clinic. Pediatr Dermatol 1983;1(2): 157-64.

5. McMahon P, Goddard D, Frieden IJ. Pediatric dermatology inpatient consultations: a retrospective study of 427 cases. J Am Acad Dermatol 2013;68(6):926-31.

6. Storan ER, McEvoy MT, WetterDA, et al. Pediatrichospital dermatology: experience with inpatient and consult services at the Mayo Clinic. Pediatr Dermatol 2013;30(6):433-7.

7. Peñate $\mathrm{Y}$, Borrego L, Hernández $\mathrm{N}$, et al. Pediatric dermatology consultations: a retrospective analysis of inpatient consultations referred to the dermatology service. Pediatr Dermatol 2012;29(1):115-8.

8. Harper J, Oranje A, Prose NS. Textbook of Pediatric Dermatology.2nd ed. Malden: Blackwell Publishing;2006.

9. Kirsner RS, Yang DG, Kerdel FA. Dermatologic disease accounts for a large number of hospital admissions annually. J Am Acad Dermatol 1999;41(6):970-3.
10. Ferrer AP, Sucupira AC, Grisi SJ. Causes of hospitalization among children ages zero to nine years old in the city of São Paulo, Brazil. Clinics (Sao Paulo) 2010;65(1):35-44.

11. Castro MSM, Travassos C, Carvalho MS. Analysis of hospital admissions associated factors in Brazil. Ciên Saúde Colet 2002;7(4):795-811.

12. Afsar FS. Pediatric dermatology in practice: spectrum of skin diseases and approach to patients at a Turkish pediatric dermatology center. Cutan Ocul Toxicol 2011;30(2):138-46.

13. Williams HC. Epidemiology of skin diseases. In: Burns DA, Breatnach SM, Cox NH, eds. Rook's Textbook of Dermatology. 7th ed Oxford: Blackwell Science; 2004.p.161-2.

14. Wenk C, Itin PH. Epidemiology of pediatric dermatology and allergology in the region of Aargau, Switzerland. Pediatr Dermatol 2003;20(6):482-7.

15. Karthikeyan K, Thappa DM, Jeeavankumar B. Pattern of pediatric dermatoses in a referral center in South India. Indian Pediatr 2004;41(4):373-7.

16. Mancusi S, Festa Neto C. Inpatient dermatological consultations in a university hospital. Clinics (Sao Paulo) 2010;65(9):851-5.

17. Nahass GT. Inpatient dermatology consultation. Dermatol Clin 2000;18(3):533-42.

18. Itin PH. Dermatologic consultations in the hospital ward: the skin, interdisciplinary organ. Dermatology 2009;219(3):193-4.

19. BenSaifGA, AlShebabSA. Pattern of childhood dermatoses at a teaching hospital of Saudi Arabia. Int J Health Sci (Qassim) 2008;2(2):63-74. 


\section{ANNEX}

\section{Groups of skin diseases and consult diagnoses and their frequencies in pediatric dermatology inpatient consultations}

\section{Groups of skin diseases}

Inpatients (n) $\quad(\%)$

Allergic skin diseases

Atopic dermatitis

Unclassified eczema

Papular urticarial

Irritant contact dermatitis

Diaper dermatitis

Seborrheic dermatitis

Maculopapular drug eruption

Urticaria

Allergic contact dermatitis

Xeroderma

Pityriasis alba

Fixed drug eruption

Nummular eczema

Dyshidrotic eczema

Perioral dermatitis

Phototoxic contact dermatitis

Juvenile plantar dermatosis

Cheilitis

Solar dermatitis

Infectious diseases

Pyoderma (unclassified)

Herpes simplex virus infection

Impetigo

Varicella

Tinea capitis

Viral wart

Cellulitis

Viral exanthem caused by enteroviruses

Tinea corporis

Tinea pedís

Molluscum contagiosum

Candidiasis

Scabies

Pediculosis capitis

Cutaneous leishmaniasis

Herpes zoster

Echtyma

Staphylococcal scalded skin syndrome

Systemic disorders with

cutaneous manifestations

Henoch-Schönlein vasculitis

Petechia/purpura

Urticarial vasculitis

Purpura fulminans

Job's syndrome

Kawasaki's disease

Livedo reticularis

Behcet's disease

Dermatomyositis

Localized scleroderma

Genodermatoses

Epidermolysis bullosa

Ichtyosis

Palmoplantar keratoderma

Collodion baby

Ectodermal dysplasia

\begin{tabular}{cc} 
Inpatients (n) & $\mathbf{( \% )}$ \\
\hline 254 & $(\mathbf{4 7 . 1 \% )}$ \\
40 & $(7.4 \%)$ \\
40 & $(7.4 \%)$ \\
29 & $(5.4 \%)$ \\
28 & $(5.2 \%)$ \\
24 & $(4.5 \%)$ \\
20 & $(3.7 \%)$ \\
18 & $(3.3 \%)$ \\
16 & $(3.0 \%)$ \\
11 & $(2.0 \%)$ \\
6 & $(1.1 \%)$ \\
5 & $(0.9 \%)$ \\
5 & $(0.9 \%)$ \\
3 & $(0.5 \%)$ \\
2 & $(0.4 \%)$ \\
2 & $(0.4 \%)$ \\
2 & $(0.4 \%)$ \\
1 & $(0.2 \%)$ \\
1 & $(0.2 \%)$ \\
1 & $(0.1 \%)$ \\
$\mathbf{7 9}$ & $(\mathbf{1 4 . 7 \% )}$ \\
17 & $(3.2 \%)$ \\
12 & $(2.3 \%)$ \\
9 & $(1.6 \%)$ \\
7 & $(1.3 \%)$ \\
7 & $(1.3 \%)$ \\
7 & $(1.3 \%)$ \\
4 & $(0.7 \%)$ \\
3 & $(0.5 \%)$ \\
2 & $(0.4 \%)$ \\
2 & $(0.4 \%)$ \\
2 & $(0.4 \%)$ \\
1 & $(0.2 \%)$ \\
1 & $(0.2 \%)$ \\
1 & $(0.2 \%)$ \\
1 & $(0.2 \%)$ \\
1 & $(0.2 \%)$ \\
1 & $(0.2 \%)$ \\
1 & $(0.2 \%)$ \\
&
\end{tabular}

Groups of skin diseases

Inpatients (n) (\%)

Rothmund-Thomson syndrome

$(0.4 \%)$

Tuberous sclerosis

$(0.4 \%)$

Incontinentia pigmenti

$(0.4 \%)$

Papillon Lefévre syndrome

$(0.4 \%)$

Miscellaneous

$(6.1 \%)$

Erythema toxicum neonatorum

$(0.7 \%)$

Mastocytosis

$(0.5 \%)$

Aphtous stomatitis

$(0.5 \%)$

Leukoplakia

Decubitis ulcer

Neurodegenerative disease

Juvenile xanthogranuloma

Langerhans cell histiocytosis

Pigmented purpuric dermatosis

Keloid scar

$(0.5 \%)$

$(0.5 \%)$

$(0.2 \%)$

$(0.2 \%)$

$(0.2 \%)$

$(0.2 \%)$

$(0.2 \%)$

$(0.2 \%)$

$(0.2 \%)$

Striae

Calcinosis cutis

Foreign body granuloma

Subcutaneous tumor

$(0.2 \%)$

$(0.2 \%)$

$(0.2 \%)$

$(0.2 \%)$

$(0.2 \%)$

$(0.2 \%)$

$(0.2 \%)$

$(0.2 \%)$

$(0.2 \%)$

$(0.2 \%)$

Transient pustular melanosis

$(4.4 \%)$

sebaceous and eccrine glands

$(0.9 \%)$

Nail dystrophy

Acne

Scarring alopecia

Alopecia areata

Miliaria

Pachyonichia congénita

Telogen effluvium

Infantile acne

Hair shaft abnormality

Hypertrichosis

Erythemas and blistering disorders

Erythema multiforme

Erythema nodosum

Erythroderma

Stevens-Johnson syndrome

Linear IgA dermatosis

Erythema annulare centrifugum

$(0.7 \%)$

$(0.7 \%)$

$(0.5 \%)$

$(0.4 \%)$

$(0.4 \%)$

$(0.2 \%)$

$(0.2 \%)$

$(0.2 \%)$

$(0.2 \%)$

$(3.9 \%)$

$(1.5 \%)$

$(0.7 \%)$

$(0.7 \%)$

$(0.4 \%)$

$(0.4 \%)$

$(0.2 \%)$

Disorders of pigmentation/melanocyte and epidermal naevi

Vitiligo

Congenital melanocytic nevus

Postinflammatory hypopigmentation

Linear epidermal nevus

$(0.5 \%)$

$(0.4 \%)$

$(0.4 \%)$

$(0.4 \%)$

$(0.2 \%)$

Nevus depigmentosus

Acquired melanocytic nevus

$(0.2 \%)$

$(0.2 \%)$

Postinflammatory hyperpigmentation

Freckling (ephelides) 
e384 / Arch Argent Pediatr 2017;115(6):e377-e384 / Original article

\begin{tabular}{|c|c|c|c|c|c|}
\hline Groups of skin diseases & Inpatients (n) & $(\%)$ & Groups of skin diseases & Inpatients (n) & $(\%)$ \\
\hline Halo nevus & 1 & $(0.2 \%)$ & Pityriasis rosea & 1 & $(0.2 \%)$ \\
\hline Papulosquamous disorders & 15 & $(2.8 \%)$ & Lichen planus & 1 & $(0.2 \%)$ \\
\hline Psoriasis & 8 & $(1.5 \%)$ & Vascular and lymphatic anomalies & 7 & $(1.3 \%)$ \\
\hline Pityriasis lichenoides & 4 & $(0.7 \%)$ & Port-wine stain & 5 & $(0.9 \%)$ \\
\hline \multirow[t]{2}{*}{ Pityriasis rubra pilaris } & 1 & $(0.2 \%)$ & Hemangioma & 2 & $(0.4 \%)$ \\
\hline & & & Total & 539 & $(100 \%)$ \\
\hline
\end{tabular}

